TRANSACTIONS OF THE

AMERICAN MATHEMATICAL SOCIETY

Volume 319, Number 1, May 1990

\title{
ISOMORPHISM UNIVERSAL VARIETIES OF HEYTING ALGEBRAS
}

\author{
M. E. ADAMS, V. KOUBEK AND J. SICHLER
}

\begin{abstract}
A variety $\mathbf{V}$ is group universal if every group $G$ is isomorphic to the automorphism group $\operatorname{Aut}(A)$ of an algebra $A \in \mathbf{V}$; if, in addition, all finite groups are thus representable by finite algebras from $\mathbf{V}$, the variety $\mathbf{V}$ is said to be finitely group universal. We show that finitely group universal varieties of Heyting algebras are exactly the varieties which are not generated by chains, and that a chain-generated variety $\mathbf{V}$ is group universal just when it contains a four-element chain. Furthermore, we show that a variety $\mathbf{V}$ of Heyting algebras is group universal whenever the cyclic group of order three occurs as the automorphism group of some $A \in \mathbf{V}$. The results are sharp in the sense that, for every group universal variety and for every group $G$, there is a proper class of pairwise nonisomorphic Heyting algebras $A \in \mathbf{V}$ for which $\operatorname{Aut}(A) \cong G$.
\end{abstract}

\section{INTRODUCTION}

A well-known result of Garret Birkhoff [3] states that every group is isomorphic to the automorphism group of a distributive lattice. On the other hand, McKenzie and Monk [14] demonstrated that Boolean algebras fail to represent all groups in this way. Heyting algebras are a natural generalization of Boolean algebras and, as one of the results of [1] implies, they form an isomorphism universal variety, that is, a variety whose isomorphisms form a category which contains any category consisting of invertible morphisms as a full subcategory. In particular, for every group $G$ there is a proper class $\mathbf{C}$ of nonisomorphic Heyting algebras such that $\operatorname{Aut}(H)$ is isomorphic to $G$ for every $H \in \mathbf{C}$.

The nontrivial varieties of Heyting algebras form an uncountable $(0,1)$ lattice (see Jankov [11]) for which the variety of Boolean algebras is the zero and the variety of all Heyting algebras is the unit. The above-mentioned contrast between Boolean algebras and Heyting algebras leads naturally to the question of characterizing all isomorphism universal varieties of Heyting algebras. The present paper provides such a characterization.

Recall that an algebra $(H ; \vee, \wedge, \rightarrow, 0,1)$ of type $(2,2,2,0,0)$ is a Heyting algebra if $(H ; \vee, \wedge, 0,1)$ is a distributive $(0,1)$-lattice and $\rightarrow$ is

Received by the editors February 15, 1988 and, in revised form, August 15, 1988.

1980 Mathematics Subject Classification (1985 Revision). Primary 06D20, 18B15; Secondary: 03G25, 08A35.

The support of NSERC of Canada is gratefully acknowledged by the second and third authors.

(C) 1990 American Mathematical Society $0002-9947 / 90 \$ 1.00+\$ .25$ per page 
the operation of relative pseudocomplementation defined by the requirement that $z \leq x \rightarrow y$ just when $z \wedge x \leq y$.

Let $\mathbf{K}$ denote the variety of Heyting algebras determined by the identity $(x \rightarrow y) \vee(y \rightarrow x)=1$. As shown by Hecht and Katriňák [9] (cf. Balbes and Dwinger [2]), the lattice of subvarieties of $\mathbf{K}$ is the $(\omega+1)$-chain

$$
\mathbf{K}_{1} \subset \mathbf{K}_{2} \subset \cdots \subset \mathbf{K}_{n} \subset \cdots \subset \mathbf{K}
$$

in which $\mathbf{K}_{1}$ is the trivial variety, $\mathbf{K}_{n}$ is generated by the $n$-element chain $C_{n}$ for every $n \geq 2$, and $\mathbf{K}$ is the variety generated by all (finite) chains. The defining identity of $\mathbf{K}_{n}$ is $\left(x_{1} \rightarrow x_{2}\right) \vee\left(x_{2} \rightarrow x_{3}\right) \vee \cdots \vee\left(x_{n} \rightarrow x_{n+1}\right)=1$.

We prove the two results below.

Theorem 1.1. The following statements are equivalent for any variety $\mathbf{V}$ of Heyting algebras:

(i) $\mathbf{V}$ fails the identity $\left(x_{1} \rightarrow x_{2}\right) \vee\left(x_{2} \rightarrow x_{3}\right) \vee\left(x_{3} \rightarrow x_{4}\right)=1$,

(ii) the cyclic group of order three is isomorphic to the automorphism group of an algebra from $\mathbf{V}$,

(iii) $\mathbf{V}$ is isomorphism universal.

In particular, a chain-generated variety of Heyting algebras is isomorphism universal if and only if it contains the variety $\mathbf{K}_{4}$ while all varieties not contained in $\mathbf{K}$ are isomorphism universal.

As noted earlier, every group $G$ occurs as the automorphism group of an algebra from a given isomorphism universal variety. The second result provides additional information about the size of Heyting algebras representing a given finite group $G$.

Theorem 1.2. Let $\mathbf{V}$ be an isomorphism universal variety of Heyting algebras and let $G$ be a finite group. Then

(i) any variety $\mathbf{V}$ which fails the identity $(x \rightarrow y) \vee(y \rightarrow x)=1$ contains a finite algebra $A$ with $\operatorname{Aut}(A) \cong G$,

(ii) the variety $\mathbf{K}$ defined by the identity $(x \rightarrow y) \vee(y \rightarrow x)=1$ contains $a$ countably infinite algebra $A$ with $\operatorname{Aut}(A) \cong G$,

(iii) if the order of $G$ is odd then every $A \in \mathbf{K}$ with $\operatorname{Aut}(A) \cong G$ is infinite.

The paper is subdivided into sections dealing respectively with elements of Priestley's duality applicable to varieties of Heyting algebras and their classification, with isomorphism universal varieties not generated by chains, the proof of isomorphism universality of the largest chain generated variety $\mathbf{K}$, and of its smallest group universal subvariety $\mathbf{K}_{4}$.

Throughout the paper, we use Priestley's duality for distributive lattices [16, 17].

\section{VARIETIES GENERATED BY CHAINS}

We begin with a brief review of fundamentals of Priestley's duality. 
A triple $(X, \tau, \leq)$ is an ordered space if $\leq$ is a partial ordering and $\tau$ is a topology on the set $X$. A set $D \subseteq X$ is decreasing if $x \leq d \in D$ implies $x \in D$; an increasing set is defined dually. An ordered space is totally order disconnected if for every $x, y \in X$ with $x \nsupseteq y$ there exists a clopen decreasing set $D$ containing $x$ and not containing $y$. A compact totally order disconnected ordered space will be called a Priestley space. The category $\mathbf{P}$ of all continuous order preserving mappings between Priestley spaces is dually isomorphic to the variety of all distributive $(0,1)$-lattices $[16,17]$. Elements of the distributive lattice corresponding to a Priestley space $(X, \tau, \leq)$ are exactly all clopen decreasing subsets of $X$, while each lattice homomorphism is the inverse image map $f^{-1}$ of a morphism $f$ from $\mathbf{P}$.

The claim below (cf. Priestley's survey [18]) describes the restriction of Priestley's duality to Heyting algebras. Recall that $C$ is a convex subset of a poset $Y$ if $c, d \in C$ and $c \leq y \leq d$ imply $y \in D$. Let $[Z)$ denote the order filter of $Y$ generated by $Z \subseteq Y$ and, dually, let $(Z]$ be the smallest order ideal of $Y$ containing $Z$.

Lemma 2.1 [18]. A Priestley space $(X, \tau, \leq)$ is an $h$-space, that is, the dual of a Heyting algebra, if and only if $[C)$ is clopen for every clopen convex $C \subseteq X$. If $A$ and $B$ are clopen decreasing sets representing the algebra elements $a$ and $b$ respectively, then $a \rightarrow b$ is represented by the (clopen decreasing) set $X \backslash[A \backslash B)$.

$A$ continuous order preserving mapping $f$ between such spaces is an h-map, that is, a mapping representing a Heyting algebra homomorphism if and only if $(f(x)]=f((x])$ for every element $x$ of the domain space of $f$.

Thus any finite distributive lattice is the underlying lattice of a Heyting algebra and any invertible $\mathbf{P}$-morphism of $h$-spaces is an $h$-map.

Of particular interest are the five-element Heyting algebra $H=\{0, p, q, u$, 1\} with incomparable $p, q<u$ and the six-element algebra $H^{\prime}=\{0, z, p, q$, $u, 1\}$ whose elements $p, q$ are again incomparable and satisfy $z<p, q$ and $p, q<u$.

Lemma 2.2. Let $\mathbf{V}$ be a variety of Heyting algebras. Then $\mathbf{V}$ either satisfies the identity $(x \rightarrow y) \vee(y \rightarrow x)=1$ and is generated by chains or else fails this identity and contains $\mathbf{H}=\operatorname{Var}(H)$ or $\mathbf{H}^{\prime}=\operatorname{Var}\left(H^{\prime}\right)$.

Proof. Assume that $\mathbf{V}$ satisfies the identity $(x \rightarrow y) \vee(y \rightarrow x)=1$. It is well known that every subdirectly irreducible Heyting algebra $S$ has a join irreducible unit 1; thus if $S \in \mathbf{V}$ and $x, y \in S$ satisfy $x \leq \leq y$, then $x \rightarrow y<1$. Hence $y \rightarrow x=1$, from which $y \leq x$ is obtained. Thus $\mathbf{V}$ is generated by chains.

Conversely, let $u=(a \rightarrow b) \vee(b \rightarrow a)<1$ in some algebra $A \in \mathbf{V}$. Set $p=a \rightarrow b$ and $q=b \rightarrow a$. Either $p \wedge q=0$ in $A$ or $p \wedge q=z>0$. Using 2.1 or a direct calculation, it is routine to verify that the distributive sublattices $\{0, p, q, u, 1\},\{0, z, p, q, u, 1\}$ of $A$ are closed under relative complementation in $A$. Thus $\mathbf{V}$ contains at least one of the subdirectly irreducible Heyting algebras $H, H^{\prime}$. 
Let $(X, \tau, \leq)$ be an $h$-space. If $a, b \in X$ are incomparable then there exist clopen decreasing sets $A$ and $B$ such that $a \in A \backslash B$ and $b \in B \backslash A$. Since $A \rightarrow B=X \backslash[A \backslash B)$ and $B \rightarrow A=X \backslash[B \backslash A)$, the equation $(A \rightarrow B) \vee(B \rightarrow$ $A)=1$ holds if and only if $[A \backslash B) \cap[B \backslash A)=\varnothing$. This shows that the Heyting algebra represented by $(X, \tau, \leq)$ satisfies the identity $(y \rightarrow z) \vee(z \rightarrow y)=1$ if and only if $(x]$ is a chain for every $x \in X$, that is, just when every order component of $(X, \leq)$ is a tree. Since every subdirectly irreducible Heyting algebra $A$ has a join irreducible unit, $A \backslash\{1\}$ is its largest prime ideal; since $(X, \leq)$ is isomorphic to the poset of all prime ideals of the associated Heyting algebra, the $h$-space of a subdirectly irreducible algebra $A \in \mathbf{K}$ is a chain.

Recall that $x \rightarrow y=1$ whenever $x \leq y$; if $C$ is a chain and $x, y \in C$ then also $x \rightarrow y=y$ for $x>y$. Hence the identity

$$
\left(x_{1} \rightarrow x_{2}\right) \vee\left(x_{2} \rightarrow x_{3}\right) \vee \cdots \vee\left(x_{n} \rightarrow x_{n+1}\right)=1
$$

fails in $C$ if and only if $x_{1}>x_{2}>\cdots>x_{n+1}$. Equivalently, this identity holds in a chain $C$ just when $C$ has at most $n$ elements. Hence the subdirectly irreducible members of a nontrivial variety $\mathbf{K}_{n}$ are chains with $k \leq n$ elements. Since every algebra $A \in \mathbf{K}_{n}$ can be embedded into a power of $C_{n}$, for any $h$-space $(X, \tau, \leq)$ dual to an algebra in $\mathbf{K}_{n}$, the poset $(X, \leq)$ is a disjoint union of trees whose chains have at most $n-1$ elements. Conversely, such an $h$-space can be covered by images of one-to-one $h$-maps of $k$-element chains with $k \leq n-1$, and thus it represents an algebra in $\mathbf{K}_{n}$.

Lemma 2.3. The cyclic group of order three is not isomorphic to the automorphism group of any algebra $A \in \mathbf{K}_{\mathbf{3}}$.

Proof. Let $\alpha$ be the dual of an automorphism of $A$ of order three, and let $b \in X$ satisfy $\alpha(b) \neq b$.

First, if $b$ is a minimal element of $X$, then $\alpha(b)$ and $\alpha^{2}(b)$ are also minimal, and there exists a clopen decreasing set $B \subseteq X$ such that $b \in B$ and $\alpha(b), \alpha^{2}(b) \in X \backslash B$. The set $C=[B)$ is clopen since $X$ is an $h$-space. For every $c \in C$, the order ideal $(c]$ is a chain whose minimal element lies in $B$, so that $C$ is also decreasing. Clearly $b \in C$; if $b \in \alpha(C) \cup \alpha^{2}(C)$ then $\alpha^{2}(b) \in C$ or $\alpha(b) \in C$, contrary to the choice of $B$. Therefore the clopen set $D=C \backslash\left(\alpha(C) \cup \alpha^{2}(C)\right)$ contains $b$, and is both increasing and decreasing. Clearly $D \cap \alpha(D)=\varnothing$. Define $\beta: X \rightarrow X$ by $\beta(d)=\alpha(d)$ and $\beta(\alpha(d))=d$ for $d \in D$, and by $\beta(x)=x$ for all other $x \in X$. It is now easily seen that $\beta$ is an order preserving homeomorphism of order two.

Secondly, assume that $\alpha(m)=m$ for all minimal $m \in X$. Since all chains of $X$ have at most two elements, $b$ must be maximal. There exists a clopen increasing set $C$ containing $b$ and such that $\alpha(b), \alpha^{2}(b) \in X \backslash C$. As before, the set $D=C \backslash\left(\alpha(C) \cup \alpha^{2}(C)\right)$ is clopen, nonvoid, and disjoint from $\alpha(D)$. By the hypothesis, $D$ and $\alpha(D)$ consist entirely of maximal elements of $X$. The mapping $\beta$ defined as in the previous case is a homeomorphism of order two. 
To see that $\beta$ preserves order, note that $x<d$ if and only if $x<\alpha(d)$ for any $d \in D$, and that $\beta(x)=x=\alpha(x)$ for all such $x \in X$.

This shows that no proper subvariety of $\mathbf{K}_{4}$ is isomorphism universal.

To demonstrate 1.2(iii), we show that any finite algebra in $\mathbf{K}$ whose automorphism group is nontrivial must have an automorphism of order two. To see this, recall that the Priestley space $P$ of a finite algebra $A \in \mathbf{K}$ is a discrete space whose order is a disjoint union of trees. If $\alpha$ is the dual of a nontrivial automorphism of $A$, let $p \in P$ be any element of $P$ which is minimal with respect to the requirement that $\alpha(p) \neq p$. The order filters $[p)$ and $[\alpha(p))$ are thus disjoint, and $\alpha$ maps $[p)$ isomorphically onto $[\alpha(p))$. Also, $\alpha(x)=x$ for any $x<p$ and, consequently, $x=\alpha(x)<\alpha(p)$. It is now easy to see that the mapping $\gamma: P \rightarrow P$ defined by $\gamma(y)=\alpha(y)$ for $y \in[p), \gamma(y)=\alpha^{-1}(y)$ for $y \in[\alpha(p))$, and $\gamma(x)=x$ for all other $x \in P$ is an order automorphism of the poset $P$. Hence any algebra in $\mathbf{K}$ representing a group with no elements of order two must be infinite.

\section{VARIETIES NOT CONTAINED IN $\mathbf{K}$}

This section shows that any variety of Heyting algebras failing the identity $(x \rightarrow y) \vee(y \rightarrow x)=1$ is isomorphism universal. Heyting algebras of the isomorphism universal class will be described through Priestley's duality.

Let $(X, E)$ be an undirected graph with no loops, that is, let $E$ be a set of two-element subsets of the set $X$. A mapping $f: X \rightarrow X^{\prime}$ is a graph morphism of $(X, E)$ into $\left(X^{\prime}, E^{\prime}\right)$ if $\{f(x), f(y)\} \in E^{\prime}$ for every $\{x, y\} \in E$.

Frucht [7] has shown that every group is isomorphic to the group of all invertible endomorphisms of some graph. The reader is referred to [19] for the fact that the category of all invertible homomorphisms of connected undirected graphs is isomorphism universal.

Since a category $\mathbf{C}$ of invertible morphisms is isomorphism universal if and only if its dual $\mathbf{C}^{\mathrm{opp}}$ is, to prove the isomorphism universality of a class $\mathbf{V}$ of Heyting algebras, it suffices to show the isomorphism universality of the class of $h$-spaces representing algebras from $\mathbf{V}$.

Given a connected undirected graph $G=(X, E)$, let $F(X, E)$ denote the poset defined on the (disjoint) union $Y=X \cup E$ by $y \leq y^{\prime}$ if and only if $y=y^{\prime}$ or $y \in y^{\prime}$. by

For any invertible graph morphism $f: G \rightarrow G^{\prime}$ define $F(f): F(G) \rightarrow F\left(G^{\prime}\right)$

$$
F(f)(x)=f(x) \text { for all } x \in X,
$$

and

$$
F(f)(\{x, y\})=\{f(x), f(y)\} \text { for all }\{x, y\} \in E .
$$

Lemma 3.1 [15 or 20]. A mapping $h: F(G) \rightarrow F\left(G^{\prime}\right)$ is invertible order preserving if and only if $h=F(f)$ for some invertible graph morphism $f: G \rightarrow G^{\prime}$. 
For a graph $G=(X, E)$ and $x \in X$, denote $E(x)=\{e \in E: x \in e\}$. Let $\beta(E)$ be the Stone-Čech compactification of the discrete set $E$, that is, the set of all ultrafilters on $E$ in which the principal ultrafilters are represented by their generating elements. For any $g: E \rightarrow E^{\prime}$, let $\beta(g)$ denote the unique continuous extension of $g$.

We define an ordered topological space $\Phi(G)$ as follows.

For any finite graph $G$, let $\Phi(G)=F(G)$ with the discrete topology.

If $X$ is infinite then the connectedness of $G$ implies that $E$ is also infinite; in this case we define $\Phi(G)$ on the set

$$
Z=X \cup \beta(E) \cup\{0\} .
$$

Hence $Z$ contains the underlying set $X \cup E$ of $F(G)$. The order of $\Phi(G)$ is defined as follows: $z \leq z^{\prime}$ if and only if one of the clauses below applies:

$$
\begin{aligned}
& z=z^{\prime} \\
& z, z^{\prime} \in F(G) \text { and } z \leq z^{\prime} \text { in } F(G), \\
& z \in X, z^{\prime} \in \beta(E) \backslash E \text { and } E(z) \in z^{\prime}, \\
& z=0 \text { and } z^{\prime} \in \beta(E) \backslash E .
\end{aligned}
$$

The topology of $\Phi(G)$ is the union of the compact topology $\beta(E)$ and the one-point compactification $X \cup\{0\}$ of the discrete set $X$.

The compact ordered space $\Phi(G)$ is thus a disjoint union of its maximal and minimal elements, and $(z] \backslash\{z\}$ has at most two elements for every $z \in Z$. Clopen points of $\Phi(G)$ form the subset $X \cup E$ of $Z$.

Lemma 3.2. The subsets $(z]$ and $[z)$ of $\Phi(G)$ are clopen for every $z \in X \cup E$. Proof. Since there is nothing to prove when $G$ is finite, assume $G$ to be infinite.

If $z=\{x, y\} \in E$ then $(z]=\{x, y, z\}$ and $[z)=\{z\}$ are subsets of $X \cup E$, hence clopen.

For $z \in X$ the set $(z]=\{z\}$ is clopen. For $[z)=\{z\} \cup\{u \in \beta(E): E(z) \in$ $u\}$, the singleton $\{z\}$ is clopen; since $V \subset \beta(E)$ is clopen just when $V=\{u \in$ $\beta(E): W \in \mathcal{U}\}$ for some $W \subseteq E$, the set $[z)$ is clopen again.

Lemma 3.3. $\Phi(G)$ is an $h$-space for any graph $G$.

Proof. Again, it suffices to consider an infinite $\Phi(G)$.

The space $\Phi(G)$ is clearly compact. To show that $\Phi(G)$ is totally order disconnected, select $z \not \leq w$ in $\Phi(G)$.

If either one of these elements lies in $X \cup E$, apply 3.2 .

Secondly, for $z, w \in \beta(E) \backslash E$ there exists a clopen set $V \subseteq \beta(E)$ such that $z \in V$ and $w \notin V$. The set $V$ is increasing and clopen in $\Phi(G)$. Thus $\Phi(G)$ is totally order disconnected.

Since minimal elements of $\Phi(G)$ form a clopen set and all other members of $\Phi(G)$ are maximal, to see that $\Phi(G)$ is an $h$-space we need only consider $[C)$ for clopen sets $C$ of minimals.

Any clopen $C \subseteq X$ is finite, and $[C$ ) is clopen by 3.2 in this case. If $0 \in C$ then $X \backslash C$ is finite, and $\Phi(G) \backslash[C)$ is a finite subset of $X \cup E$, so that $[C)$ is clopen again. 
The $h$-space $A$ corresponding to the generating algebra $H$ of the variety $\mathbf{H}$ is the three-element discrete space $\{a, b, c\}$ in which $a, b<c$. It is easy to see that the images of $h$-maps $g: A \rightarrow \Phi(G)$ cover $\Phi(G)$; thus $\Phi(G)$ is a subdirect power of $H$.

For any invertible graph morphism $f: G \rightarrow G^{\prime}$, define $\Phi(f): \Phi(G) \rightarrow \Phi\left(G^{\prime}\right)$ as follows.

For finite $G$ and $G^{\prime}$ set $\Phi(f)=F(f)$; when $G$ and $G^{\prime}$ are infinite, then

$$
\Phi(f)(z)=\left\{\begin{array}{ll}
F(f)(z) & \text { for all } z \in F(G), \\
\beta(F(f) \uparrow E)(z) & \text { for all } z \in \beta(E) \backslash E,
\end{array} \quad \Phi(f)(0)=0 .\right.
$$

It is easy to see that $\Phi(f)$ thus defined is an invertible $h$-map in either case.

Lemma 3.4. For each invertible h-map $h: \Phi(G) \rightarrow \Phi\left(G^{\prime}\right)$ there exists a unique invertible graph morphism $f: G \rightarrow G^{\prime}$ such that $h=\Phi(f)$.

Proof. When $G$ and $G^{\prime}$ are finite graphs, use Lemma 3.1.

If $G$ and $G^{\prime}$ are infinite then $h(X \cup E)=X^{\prime} \cup E^{\prime}$ since these sets are exactly the sets of open points of the respective spaces. By 3.1, there exists a unique graph morphism $f: G \rightarrow G^{\prime}$ such that $F(f)=h \uparrow F(G)$. But then $h$ and $\Phi(f)$ coincide on the dense subset $X \cup E$ of $\Phi(G)$, so that $h=\Phi(f)$.

This completes the proof of the isomorphism universality of the variety $\mathbf{H}$.

The variety $\mathbf{H}^{\prime}$ is generated by the algebra $H^{\prime}$ whose $h$-space is the extension $A^{\prime}=A \cup\{d\}$ of $A$ of which $d$ is the least element. Defining $\Phi^{\prime}(G)=\Phi(G) \cup\{d\}$ as the extension of $\Phi(G)$ by a least element $d$ which is open gives an $h$-space dual to an algebra in $\mathbf{H}^{\prime}$, for reasons similar to those used in the case of $\mathbf{H}$.

The extension $\Phi^{\prime}(f)$ of $\Phi(f)$ determined by $\Phi^{\prime}(f)(d)=d$ is an invertible $h$-map and, conversely, each invertible $h$-map between such spaces must preserve the least element $d$.

This concludes the proof of isomorphism universality of $\mathbf{H}^{\prime}$, and of Theorem 1.2(i) since any finite group is isomorphic to the group of all invertible endomorphisms of some finite graph [7].

\section{ISOMORPHISM UNIVERSALITY OF $\mathbf{K}$}

Recall that the variety $\mathbf{K}$ defined by the identity $(x \rightarrow y) \vee(y \rightarrow x)=1$ is dual to the class of all $h$-spaces whose order is a disjoint union of trees.

Recall also that a category $\mathbf{C}$ is isomorphism universal if and only if its dual $\mathbf{C}^{\text {opp }}$ is. Let $\mathbf{D}$ be the category of all digraphs $(X, R)$ and all their isomorphisms; that is, $R \subseteq X^{2}$ and an invertible mapping $f: X \rightarrow X^{\prime}$ is a morphism of $\mathbf{D}$ from $(X, R)$ to $\left(X^{\prime}, R^{\prime}\right)$ just when $(f(x), f(y)) \in R^{\prime}$ is equivalent to $(x, y) \in R$. The category $\mathbf{D}$ is isomorphism universal [19]. Thus the isomorphism universality of the variety $\mathbf{K}$ will follow once $\mathbf{D}$ is suitably represented in the category of all $h$-spaces over disjoint unions of trees.

The tree intended as a replacement for each pair $(x, y) \in R$ is defined first.

Let $\omega$ be the least infinite ordinal. Define

$$
T=\{(0, i): i \in \omega\} \cup\{(1, i): i \in \omega \text { and } i \geq 2\},
$$


and let $\leq$ be the least partial order on $T$ for which

$$
(0, i) \leq \begin{cases}(0, j) & \text { when } i, j \in \omega \text { and } i \geq j \\ (1, i) & \text { when } i \in \omega \text { and } i \geq 2\end{cases}
$$

It is easily seen that $(T, \leq)$ is a tree in which $(0, i)$ covers $(0, i+1)$ for every $i \in \omega$ and, for $i \geq 2$, each $(1, i)$ covers $(0, i)$. Set $S=\{(0, i): i \geq 1\}$, and note that $T \backslash S$ is the set of all maximal elements of $T$.

Lemma 4.1. The poset $(T, \leq)$ is automorphism free.

Proof. Any invertible order preserving mapping $f: T \rightarrow T$ preserves the number of covers of every element. Hence $f(0,1)=(0,1)$ because $(0,1)$ is the only member of $(T, \leq)$ with exactly one cover. Each $(0, i)$ with $i \geq 1$ covers only $(0, i+1)$, and $f(0, i)=(0, i)$ for all $i \geq 1$ follows inductively; thus $f$ is the identity on $S$. Members of $T \backslash S$ are maximal and cover distinct members of $S$, so that $f(t)=t$ also for all $t \in T \backslash S$.

For $k \in\{0,1\}$ set $T(k)=\{(1,2 i+k) \in T: i \geq 1\}$. These two sets are disjoint and consist of maximal elements of $T$.

Let $(X, R)$ be an object from $\mathbf{D}$. Set $E=R \times T$, and define the order of $E$ by requiring that $(r, t) \leq\left(r^{\prime}, t^{\prime}\right)$ in $E$ if and only if $r=r^{\prime}$ and $t \leq t^{\prime}$ in $T$; thus $E$ is just a disjoint union of copies $\{r\} \times T$ of $T$ with $r \in R$.

Let $(Z, \leq)=F(X, R)$ be the union of $E$ with the antichain $X$; each nontrivial order component of $Z$ is thus isomorphic to $T$, so that all order components of $(Z, \leq)$ are automorphism free. Since any invertible order preserving mapping $h: F(X, R) \rightarrow F\left(X^{\prime}, R^{\prime}\right)$ maps an order component isomorphically onto another one, there exist bijections $g: R \rightarrow R^{\prime}$ and $f: X \rightarrow X^{\prime}$ such that $h(r, t)=(g(r), t)$ for all $(r, t) \in E$ and $h(x)=f(x)$ for all $x \in X$. Conversely, any pair of bijections $f$ and $g$ defines such an order isomorphism $h: F(X, R) \rightarrow F\left(X^{\prime}, R^{\prime}\right)$.

For any $x \in X$ set $Z(x, 0)=\{((x, y), t): t \in T(0),(x, y) \in R\}, Z(x, 1)$ $=\{((y, x), t): t \in T(1),(y, x) \in R\}$, and $Z(x)=Z(x, 0) \cup Z(x, 1)$. Let $\tau$ be the collection of all subsets $A$ of $Z=F(X, R)$ such that

$$
x \in A \cap X \quad \text { implies that } Z(x) \backslash A \text { is finite. }
$$

It is easy to see that $\tau$ is a topology on $Z$ given by its open sets, and that each $x \in X$ is the point compactifying the discrete set $Z(x) \subseteq E$. The claim below readily follows.

Lemma 4.2. $A$ subset $C$ of $Z$ is clopen in $\tau$ if and only if

(a) $Z(x) \backslash C$ is finite whenever $x \in C$, and

(b) $x \in C$ whenever $C \cap Z(x)$ is infinite.

Lemma 4.3. A subset $C$ of $Z$ is compact clopen if and only if

(a) $C \cap X$ is finite,

(b) $Z(x) \backslash C$ is finite whenever $x \in C$, and 
(c) $C \backslash \bigcup\{Z(x): x \in C \cap X\}$ is a finite set.

Proof. Assume (a), (b) and (c). If $W=Z(x) \cap C$ is infinite and $x \in Z \backslash C$ then $W$ is a subset of the finite set from (c); this contradiction shows that 4.2(b) holds. Thus $C$ is clopen by 4.2. Let $S \subseteq \tau$ be such that $C \subseteq \cup S$. Using (a), select a finite $S^{\prime} \subseteq S$ such that $C \cap X \subseteq \cup S^{\prime}$. The set $Z(x) \backslash A^{\prime}$ is finite for every $x \in C \cap X$ and $x \in A^{\prime} \in S^{\prime}$; the finiteness of $C \backslash \cup S^{\prime}$ then follows from (c). Thus $C$ is also compact.

Conversely, let $C$ be compact and clopen. According to 4.2, each set $W(x)=$ $Z(x) \cup\{x\}$ is clopen and $D=Z \backslash \bigcup\{W(x): x \in X\}$ is discrete. Now (a) and (c) hold since $\{W(x): x \in X\}$ together with singletons $\{d\} \subseteq D$ form an open covering of the compact set $C$, and since $C \cap Z(x)$ is finite when $x \notin C$.

Lemma 4.4. The set $[C]$ is clopen compact for every clopen compact $C \subseteq Z$.

Proof. The set $[z]$ is clopen and finite for every $z \in E$. By 4.3(c), the set of all those $z \in C$ which are not maximal is also finite. Since all nonmaximal members of $Z$ lie in $E$, the set $[C]=C \cup\{[z): z \in C$ is nonmaximal $\}$ is clopen.

Lemma 4.5. If $z, z^{\prime} \in Z$ and $z \notin z^{\prime}$ then there exists a compact clopen increasing set $A \subseteq Z$ such that $z \in A$ and $z^{\prime} \in Z \backslash A$.

Proof. If $z \in E$ then $[z] \subseteq E$ is finite, and clopen compact by 4.3. For $z \in X$, the increasing set $A=Z(z) \backslash\left\{z^{\prime}\right\}$ is clopen compact, again by 4.3.

Altogether, $F(X, R)=(Z, \tau, \leq)$ is a totally order disconnected locally compact space. Let $\Phi(X, R)=(Z \cup\{0\}, \sigma, \leq)$ be a one-point compactification of $F(X, R)$ by the element 0 such that $0 \leq z$ for all $z \in Z$. Since all clopen compact (and/or increasing) sets of $F(X, R)$ remain clopen compact (and/or increasing) in $\Phi(X, R)$, the compact ordered space $\Phi(X, R)$ is totally order disconnected by 4.5 . Every clopen set $W$ of $\Phi(X, R)$ is either clopen in $F(X, R)$ or contains 0 ; hence [ $W$ ) is clopen, and the Priestley space $\Phi(X, R)$ represents a Heyting algebra. The description of clopen compact subsets of $F(X, R)$ given in 4.3 implies that the cardinality of the Heyting algebra represented by $\Phi(X, R)$ is $\max \left(\aleph_{0}, \operatorname{card}(X)\right)$.

For any graph isomorphism $f:(X, R) \rightarrow\left(X^{\prime}, R^{\prime}\right)$ define $\Phi(f): \Phi(X, R) \rightarrow$ $\Phi\left(X^{\prime}, R^{\prime}\right)$ by

$$
\begin{aligned}
\Phi(f)(0) & =0, \quad \Phi(f)(x)=f(x) \quad \text { for all } x \in X, \\
\Phi(f)((x, y), t) & =((f(x), f(y)), t) \quad \text { for }(x, y) \in R \text { and } t \in T .
\end{aligned}
$$

Using earlier observations, it is easy to see that $\Phi(f)$ is an order isomorphism. To verify that $\Phi(f)$ is continuous, observe that $Z(x)=f^{-1}\left(Z^{\prime}(f(x))\right)$ and $\{0\}=\Phi(f)^{-1}(\Phi(f)(0))$. Hence $\Phi(f)$ is an $h$-map.

Lemma 4.6. $\Phi$ is a one-to-one functor of $\mathbf{D}$ into the category of isomorphisms of $h$-spaces representing algebras from $\mathbf{K}$. The Heyting algebra dual to $\Phi(X, R)$ is of cardinality $\max \left(\aleph_{0}, \operatorname{card}(X)\right)$. 
In order to conclude the proof of Theorem 1.2(ii), we must show that every isomorphism $h: \Phi(X, R) \rightarrow \Phi\left(X^{\prime}, R^{\prime}\right)$ is of the form $h=\Phi(f)$ for some isomorphism $f:(X, R) \rightarrow\left(X^{\prime}, R^{\prime}\right)$.

Since 0 is the least element in either space, $h(0)=0$ and $h$ maps $F(X, R)$ into $F\left(X^{\prime}, R^{\prime}\right)$. Earlier observations show that there exist mappings $f: X \rightarrow$ $X^{\prime}$ and $g: R \rightarrow R^{\prime}$ such that $h(x)=f(x)$ for every $x \in X$ and $h((x, y), t)=$ $(g(x, y), t) \in R^{\prime} \times T$ for every $(x, y) \in R$. It is therefore enough to show that $g(x, y)=(f(x), f(y))$ for every $(x, y) \in R$.

Let $\left(x_{0}, x_{1}\right) \in R$ and $g\left(x_{0}, x_{1}\right)=\left(u_{0}, u_{1}\right) \in R^{\prime}$. For $k \in\{0,1\}$, the set $U(k)=\left\{\left(x_{0}, x_{1}\right)\right\} \times T(k)$ is an infinite subset of the discrete set $Z\left(x_{k}, k\right)$ whose closure $\operatorname{cl}\left(Z\left(x_{k}, k\right)\right)=Z\left(x_{k}, k\right) \cup\left\{x_{k}\right\}$. Hence $\operatorname{cl}(U(k))=U(k) \cup\left\{x_{k}\right\}$; since $h$ is a homeomorphism with $h(U(k))=\left\{\left(u_{0}, u_{1}\right)\right\} \times T^{\prime}(k)$, it follows that

$$
\begin{aligned}
u_{k} & \in \operatorname{cl}\left(\left\{\left(u_{0}, u_{1}\right)\right\} \times T^{\prime}(k)\right)=\operatorname{cl}(h(U(k)))=h(\operatorname{cl}(U(k))) \\
& =h(U(k)) \cup\left\{h\left(x_{k}\right)\right\}=\left\{\left(u_{0}, u_{1}\right)\right\} \times T^{\prime}(k) \cup\left\{f\left(x_{k}\right)\right\} .
\end{aligned}
$$

Hence $u_{k}=f\left(x_{k}\right)$, that is, $g\left(x_{0}, x_{1}\right)=\left(f\left(x_{0}\right), f\left(x_{1}\right)\right)$. Therefore $f:(X, R)$ $\rightarrow\left(X^{\prime}, R^{\prime}\right)$ is a graph isomorphism and $h=\Phi(f)$ as required.

This shows the isomorphism universality of the variety $\mathbf{K}$ and thus also completes the proof of Theorem 1.2.

\section{THE VARIETY $\mathbf{K}_{4}$}

Isomorphism universality of the variety $\mathbf{K}_{4}$ requires yet another approach. We begin by constructing Stone spaces of automorphism free Boolean algebras analogous to those presented by Brenner in [4].

Definition 5.1. A poset $(T, \leq)$ is an $\omega$-tree if

(a) every principal order ideal $(t]$ of $T$ is a chain,

(b) all maximal chains of $T$ are isomorphic to $\omega+1$,

(c) the intersection of any two distinct maximal chains of $T$ is finite and nonempty,

(d) each $t \in T$ has infinitely many covers or no covers at all.

Let $T(\omega)$ denote the set of all maximal elements of an $\omega$-tree $T$, and let $T(n) \subseteq T$ consist of all elements of $T$ of a finite height $n \geq 0$. By 5.1(b), for every $t \in T$ there exists an $m \in T(\omega)$ such that $t \leq m$. It is clear that the order filter $W(t)=\{u \in T: t \leq u\}$ is an $\omega$-tree for every $t \in T \backslash T(\omega)$. For any subset $T^{\prime}$ of $T$, denote $W\left(T^{\prime}\right)=\bigcup\left\{W\left(t^{\prime}\right): t^{\prime} \in T^{\prime}\right\}$.

Let $S(t)$ stand for the set of all covers of $t$ in $T$. The uniquely determined element $r=r(T) \in T$ for which $T=W(r)$ will be called the root of $T$. For each $t \in T \backslash(T(\omega) \cup\{r(T)\})$, let $t^{-}$denote the unique element of $T$ covered by $t$. 
A subset $A$ of an $\omega$-tree $T$ is to be $\tau$-open if and only if

(i) for any $a \in A \backslash T(\omega)$ there is a finite set $F \subseteq S(a)$ such that $W(S(a) \backslash F)$ $\subseteq A$

(ii) for any $a \in A \cap T(\omega)$ there exists some $t<a$ such that $W(t) \subseteq A$.

It is easily seen that the tree space $(T, \tau)$ of any $\omega$-tree $T$ is indeed a topological space. Furthermore, $B \subseteq T$ is $\tau$-closed if and only if it satisfies the following two conditions:

(iii) if $t \in T \backslash T(\omega)$ and $\{u \in S(t): W(u) \cap B \neq \varnothing\}$ is infinite then $t \in B$, and

(iv) if $t \in T(\omega)$ and $\sup (B \cap(t])=t$ then $t \in B$.

Any "wedge" $W(t)$ with $t \in T \backslash T(\omega)$ clearly satisfies (i)-(iv) and hence is $\tau$-clopen.

For any $t \in T \backslash T(\omega)$, the clopen set $W(t)$ separates $t$ from any $u \in$ $T \backslash W(t)$. If $u \in W(t)$ is distinct from $t$, then there exists a $v \in S(t)$ such that $u \geq v$, so that the clopen set $W(v)$ separates $t$ and $u$ in this case. Finally, for distinct $t, u \in T(\omega)$, the intersection of the $(\omega+1)$-chains $(t]$ and $(u]$ is a finite nonvoid chain, so that $(t] \backslash(u] \neq \varnothing$; for any $v \in(t] \backslash(u]$, the clopen set $W(v)$ contains $t$ but not $u$. Hence $(T, \tau)$ is a totally disconnected space.

Lemma 5.2. The tree space $(T, \tau)$ of any $\omega$-tree $T$ is compact and totally disconnected.

Proof. To show that $(T, \tau)$ is compact, suppose that $C$ is an open covering of $T$.

Define $P=\left\{x \in T: x^{-} \in A \in C \Rightarrow W(x) \nsubseteq A\right\}$. If $x \in T(n) \backslash P$ for some $n \geq 1$, and if $y \in W(x) \backslash T(\omega)$ is distinct from $x$, then there exists $A \in C$ such that $x^{-} \in A$ and $W(x) \subseteq A$; from $x \leq y^{-}$we see that $y \in T \backslash P$. Hence $P$ is a decreasing subset of $\bigcup(T(n): n \geq 1)$.

If $x \in T(n)$ for some $n \geq 0$ then $P \cap S(x)$ is finite, and a finite covering $C(x) \subseteq C$ of $\{x\} \cup W(S(x) \backslash P)$ can be found. Indeed, first select $A \in C$ for which $x \in A$. Since $W(S(x) \backslash F) \subseteq A$ for some finite $F \subseteq S(x)$ and because $P \cap S(x) \subseteq F$, the set $P \cap S(x)$ is finite. Also, for every $b \in F \backslash P$ there exists an $A_{b} \in C$ such that $\{x\} \cup W(b) \subseteq A_{b}$. We set $C(x)=\{A\} \cup\left\{A_{b}: b \in F \backslash P\right\}$.

A simple induction shows that $P \cap T(n)$ is finite for each $n \geq 1$. If $P \cap$ $T(n) \neq \varnothing$ for all $n \geq 1$ then there exists an infinite chain $P^{\prime}=\left\{p_{n}: n \geq 1\right\} \subseteq P$ with $p_{n} \in T(n)$ for each $n \geq 1$. By 5.1(b), the chain $P^{\prime}$ is contained in a maximal $(\omega+1)$-chain $P^{\prime \prime}$. Let $t \in P^{\prime \prime} \cap T(\omega)$. Since $t \in A$ for some $A \in C$, from (ii) and 5.1(a) it follows that $W\left(p_{n}\right) \subseteq A$ for some $n \geq 1$. Hence $W\left(p_{n+1}\right) \subseteq A \in C$, which contradicts the definition of $P$.

Therefore $P \cap T(n)=\varnothing$ for some $n \geq 1$ and, consequently, $P$ is finite. Hence $\{C(r(T))\} \cup\{C(p): p \in P\} \subseteq C$ is a finite covering of $T$.

Let $\kappa$ be an infinite cardinal and $(X, \tau)$ a topological space. Recall that $x \in X$ is of local character $\kappa=\chi(x)$ if $\kappa$ is the least cardinal for which there 
exists a system $N(x)$ of neighbourhoods of $x$ with $\kappa=\operatorname{card}(N(x))$ and such that every open neighbourhood $A$ of $x$ contains some $N \in N(x)$. If $f$ is a homeomorphism of $(X, \tau)$ onto $\left(X^{\prime}, \tau^{\prime}\right)$ then clearly $\chi(f(x))=\chi(x)$ for every $x \in X$.

Let $(T, \tau)$ be the tree space associated with an $\omega$-tree $T$. Since any $\tau$ open set $A$ containing $a \in T(\omega)$ must contain the clopen set $W(x)$ for some $x<a$ and because $(a] \backslash\{a\}$ is an $\omega$-chain, $\chi(a)=\aleph_{0}$ for every $a \in T(\omega)$. If $a \in T(n)$ for some $n \geq 0$, let $N(a)=\{W(a) \backslash W(F): F \subseteq S(a)$ is finite $\}$. Every open set $A$ with $a \in A$ contains $W(S(a) \backslash F)$ for some finite $F \subseteq S(a)$, and hence also a member of $N(a)$. Thus $\chi(a) \leq \operatorname{card}(S(a))$. If $M(a)$ is a neighbourhood system and $B \in M(a)$, let $\varphi(B) \subseteq S(a)$ consist of all $x$ such that $W(x) \nsubseteq B$. Then every $\varphi(B)$ is finite; furthermore, $\bigcup\{\varphi(B): B \in$ $M(a)\}=S(a)$ since every $W(a) \backslash W(x)$ with $x \in S(a)$ must contain some $B \in M(a)$. The system $M(a)$ is infinite since each $S(a)$ is, and $\operatorname{card}(M(a)) \geq$ $\operatorname{card}(S(a))$ follows. As a result, $\chi(a)=\operatorname{card}(S(a))$ for every $a \in T \backslash T(\omega)$.

Lemma 5.3. If an $\omega$-tree $T$ is heterogeneous, that is, if

(a) $\aleph_{0}<\operatorname{card}(S(t))$ for each $t \in T \backslash T(\omega)$, and

(b) $\operatorname{card}(S(t)) \neq \operatorname{card}\left(S\left(t^{\prime}\right)\right)$ for distinct $t, t^{\prime} \in T \backslash T(\omega)$,

then the tree space $(T, \tau)$ has no nontrivial homeomorphism onto itself.

Proof. From previous observations we see that any homeomorphism $f$ is identical on $T \backslash T(\omega)$ and preserves $T(\omega)$. The remainder follows from the fact that $(a]$ is the closure of $(a] \backslash\{a\} \subseteq T \backslash T(\omega)$ for each $a \in T(\omega)$.

Select and fix a heterogeneous $\omega$-tree $T$ and a countably infinite set $\left\{s_{0}, s_{1}\right.$, $\left.s_{2}, \ldots\right\} \subseteq T(1)$. The subsets $W\left(s_{i}\right)$ of $T$ are heterogeneous $\omega$-trees determining pairwise disjoint compact subspaces of the tree space $(T, \tau)$ associated with $T$. Let $B_{i}$ denote the tree space given by $W\left(s_{i}\right)$ for $i \geq 0$.

Select $D=\left\{d_{i}: i=1,2, \ldots\right\} \subseteq B_{0}$ in such a way that $d_{1}=s_{0}$ and $d_{2 i}, d_{2 i+1}$ are distinct elements of $S\left(d_{i}\right)$ for each $i \geq 1$. Hence $D \subseteq B_{0}$ is a binary tree rooted at $s_{0}$ and such that $\left\{d_{j}: 2^{k} \leq j<2^{k+1}\right\} \subseteq B_{0}(k)$ for each $k \geq 1$. For every $n \geq 1$, let $C_{n}$ denote the maximal chain of $D$ whose first $n$ elements are odd-numbered and the remainder even-numbered; that is,

$$
C_{n}=\left\{d_{2^{k}-1}: 1 \leq k \leq n\right\} \cup\left\{d_{2^{k}-2^{k-n}}: k \geq n+1\right\} .
$$

Set $C_{0}=\left\{d_{2^{k}-1}: k \geq 1\right\}$. Let $z_{n}=\sup \left(C_{n}\right)$, so that $\left(z_{n}\right] \backslash B_{0}(\omega)=C_{n}$ for every $n \geq 0$. The subset $Z=\left\{z_{n}: n \geq 1\right\}$ of $B_{0}(\omega)$ is countably infinite. Set $Z^{\prime}=Z \cup\left\{z_{0}\right\}$; the sets $Z$ and $Z^{\prime}$ have the following two properties:

(a) for every $x \in B_{0} \backslash B_{0}(\omega)$ there is a finite set $F \subseteq S(x)$ such that $W(x) \cap$ $Z^{\prime} \subseteq W(F)$, and

(b) for every $y \in B_{0}(\omega) \backslash\left\{z_{0}\right\}$ there exists some $x<y$ such that $W(x) \cap$ $Z^{\prime} \subseteq\{y\}$. 
To verify (a), note that there is nothing to prove when $W(x) \cap Z^{\prime}=\varnothing$. If $z_{n} \in W(x)$ then $x=d_{i}$ for some $i \geq 1$, and it suffices to set $F=\left\{d_{2 i}, d_{2 i+1}\right\}$.

For (b), let $y \in B_{0}(\omega) \backslash\left\{z_{0}\right\}$ be arbitrary. If $W(x) \cap Z^{\prime} \neq \varnothing$ for every $x \in(y] \backslash B_{0}(\omega)$ then $(y] \backslash B_{0}(\omega)$ is a chain which is maximal in $B_{0} \backslash B_{0}(\omega)$, and also contained in $\bigcup\left(C_{n}: n \geq 0\right)$. Since the latter union has no maximal chains other than $C_{n}$ for $n \geq 0$, it follows that $(y] \backslash\{y\}=C_{n}$ for some $n \geq 1$. It now suffices to select the least even-numbered $x \in D$ occurring in $C_{n}$.

Remark 5.4. Since every $W(x)$ with $x \in B_{0} \backslash B_{0}(\omega)$ is clopen compact, (a) and (b) imply that every $b \in B_{0} \backslash\left\{z_{0}\right\}$ has a clopen compact neighbourhood $N_{b}$ such that $N_{b} \cap Z^{\prime} \subseteq\{b\}$.

To produce the initial building block of a representing construction, we define a space $(B, \beta)$ as the union of all tree spaces $B_{n}$ for $n \geq 1$ and of $B_{0} \backslash\left\{z_{0}\right\}$, in which $z_{i} \in B_{0}$ is identified with the root $s_{i}$ of $B_{i}$ for each $i \geq 1$ and no other amalgamations are made. The topology $\beta$ of $B$ is the union topology.

Lemma 5.5. Distinct points of $(B, \beta)$ are separated by disjoint clopen compact sets.

Proof. Assume $b, c \in B$ to be distinct.

From property (b), for every $i \geq 1$ there exists an $x_{i} \in B_{0} \backslash B_{0}(\omega)$ such that $W\left(x_{i}\right) \cap Z^{\prime}=\left\{z_{i}\right\}$. The set $X(i)=W\left(x_{i}\right) \cup B_{i}$ is clopen compact in $B$ and $X(i) \cap X\left(i^{\prime}\right)=\varnothing$ for distinct $i, i^{\prime} \geq 1$. Being a subspace of $(T, \tau)$, each $B_{i}$ is totally disconnected and, consequently, distinct $b, c \in \bigcup\left\{B_{i}: i \geq 1\right\}$ are separated by clopen compact disjoint sets.

Let $b \in B_{0} \backslash Z^{\prime}$. Let $N_{b}$ be a compact clopen neighborhood of $b$ disjoint from $Z^{\prime}$, as given by 5.4. Suppose $c \in B_{i}$ for some $i \geq 1$. Since $B_{0}$ is compact and totally disconnected, there exists a clopen $C_{i} \subseteq B_{0}$ such that $C_{i} \cap Z^{\prime} \subseteq\left\{z_{i}\right\}$ and $C_{i} \cap N_{b}=\varnothing$. Consequently, $N_{b}$ and $\left(X(i) \cap C_{i}\right) \cup B_{i}$ are clopen compact disjoint subsets of $B$ separating $b$ from $c$. Secondly, for $c \in B_{0} \backslash Z^{\prime}$ the conclusion follows from 5.4 and from the fact that $B_{0}$ is compact and totally disconnected.

Remark 5.6. Observe that the sets $X(i)$ from the proof of 5.5 form a disjoint clopen covering of $\bigcup\left\{B_{i}: i \geq 1\right\}$. Therefore, by 5.4, any compact subset of $B$ can intersect only finitely many subspaces $B_{i}$.

For every $i \geq 1$ select and fix a three-element subset $\left\{u_{2 i}, u_{2 i+1}, y_{i}\right\} \subseteq$ $B_{i}(\omega)$, and denote $U_{k}=\left\{u_{2 i+k}: i \geq 1\right\}$ for $k \in\{0,1\}$.

Recall also that $B_{n}=W\left(s_{n}\right)$ is a subspace of the original tree space $(T, \tau)$ for every $n \geq 0$.

Now we define an ordered topological space $(C, \tau, \leq)$ in terms of $(B, \beta)$ and countably many copies of the tree space $(T, \tau)$ as follows.

The underlying set $C$ is the disjoint union

$$
C=B \cup T \times\{1,2,3, \ldots\},
$$


and the order $\leq$ on $C$ is determined by

$$
y_{i} \leq(t, i) \text { for all } t \in T \text { and all } i \geq 1 \text {. }
$$

Let $\Sigma$ be the collection of all subsets $A$ of $C$ such that

(a) $A \cap B$ is $\beta$-clopen,

(b) $\{t \in T:(t, i) \in A\}$ is $\tau$-clopen for every $i \geq 1$,

(c) $\{(t, i): t=b \in B \cap A\} \subseteq A$ for all but finitely many $i \geq 1$, and

(d) $\{(t, i): t=b \in B \backslash A\} \cap A=\varnothing$ for all but finitely many $i \geq 1$.

The system $\Sigma$ is closed under complementation and finite intersection. Let $\gamma$ be the topology on $C$ with open basis $\Sigma$. It is easily verified that $(B, \beta)$ and each copy $T \times\{i\}$ of $(T, \tau)$ are open subspaces of $(C, \tau)$; as a result, local characters of members of these spaces remain unchanged in $(C, \gamma)$.

It is also straightforward to verify that, for every $t \in \bigcup\left\{W\left(s_{i}\right): i \geq 0\right\} \subseteq$ $T \backslash\left\{z_{0}\right\}$, the set $\{t\} \cup\{(t, i): i \geq 1\}$ is the $\gamma$-closure of $\{(t, i): i \geq 1\}$.

If $B^{\prime} \subseteq B$ is $\beta$-clopen compact and $F \subseteq\{1,2, \ldots\}$ is finite, then the set

$$
Y\left(B^{\prime}, F\right)=B^{\prime} \cup\left\{(t, i): t=b \in B^{\prime} \text { and } i \notin F\right\}
$$

is $\gamma$-clopen and compact. Moreover, by $5.6, B^{\prime}$ intersects only finitely many subspaces $B_{i}$ of $B$, so that $B^{\prime} \cap\left\{y_{1}, y_{2}, \ldots\right\}$ is finite. Thus, since

$$
\left[Y\left(B^{\prime}, F\right)\right) \backslash Y\left(B^{\prime}, F\right)=\bigcup\left\{T \times\{j\}: y_{j} \in B^{\prime}\right\}
$$

and because every $T \times\{j\}$ is $\gamma$-clopen compact, the set $\left[Y\left(B^{\prime}, F\right)\right)$ is $\gamma$-clopen compact for any $\beta$-clopen compact $B^{\prime} \subseteq B$.

Lemma 5.7. If $c, d \in C$ are such that $c \leq d$ then there exists a $\gamma$-clopen compact increasing set $K$ such that $c \in K$ and $d \in C \backslash K$.

Proof. Suppose $c \leq \mathbb{L} d$ in $C$.

When $c \in T \times\{i\}$ for some $i \geq 1$, the conclusion follows for any $d \neq c$ since $c$ is maximal in $C$ and $T \times\{i\}$ is a clopen compact subspace of maximal elements of $C$ homeomorphic to the totally disconnected space $(T, \tau)$.

For $c \in B$ choose a $\beta$-clopen compact neighbourhood $N \subseteq B$ of $c$ not containing $d$, as given by 5.5 .

If $c \in B_{0} \backslash Z^{\prime}$ then there is a $\beta$-clopen compact $N_{c} \subseteq B_{0} \backslash \bigcup\left\{B_{i}: i \geq 1\right\}$ by the definition of $B$ and by 5.4. Then $c \in B^{\prime}=N_{c} \cap N \subseteq B$, and $B^{\prime}$ is a $\beta$-clopen and compact subset of $B$ disjoint from $\left\{d, y_{1}, y_{2}, \ldots\right\}$. Hence $Y\left(B^{\prime}, \varnothing\right)$ is a $\gamma$-clopen compact increasing neighbourhood of $c$. If $d \in Y\left(B^{\prime}, \varnothing\right)$ then $d \in T \times\{j\}$ for some $j \geq 1$, and $K=Y\left(B^{\prime},\{j\}\right)$ is a compact clopen increasing set as required.

Suppose now that $c \in B_{i}$ for some $i \geq 1$.

If $c \neq y_{i}$, by 5.5 there is a compact clopen $M \subseteq B$ such that $c \in M \subseteq B_{0} \cup B_{i}$ and $y_{i} \in B \backslash M$; hence $B^{\prime}=N \cap M$ is a compact $\beta$-clopen subset of $B$ disjoint from $\left\{d, y_{1}, y_{2}, \ldots\right\}$. As before, the neighbourhood $Y\left(B^{\prime}, \varnothing\right)$ of $c$ is $\gamma$ clopen, compact and increasing, and so is any $Y\left(B^{\prime},\{j\}\right)$. 
If $c=y_{i}$ then there exists a $\beta$-clopen set $M$ with $c \in M \subseteq B_{i}$; the set $B^{\prime}=$ $N \cap M$ is a $\beta$-clopen compact subset of $B$ disjoint from $\left\{d, y_{1}, y_{2}, \ldots, y_{i-1}\right.$, $\left.y_{i+1}, \ldots\right\}$. Again, the neighbourhood $Y\left(B^{\prime}, \varnothing\right)$ of $c$ is $\gamma$-clopen compact increasing, and so is each $Y\left(B^{\prime},\{j\}\right)$ with $j \neq i$.

Lemma 5.8. If $K$ is a $\gamma$-clopen compact subset of $C$ then $[K]$ is $\gamma$-clopen and compact.

Proof. Since $B$ is $\gamma$-closed, $K \cap B$ is compact in $B$. Hence the set $K \cap$ $\left\{y_{1}, y_{2}, \ldots\right\}$ is finite by 5.6 , and the conclusion follows from $[K) \backslash K=$ $\bigcup\left\{T \times\{i\}: y_{i} \in K\right\}$ and the fact that each $T \times\{i\}$ is $\gamma$-clopen compact.

The class $2 \mathrm{~A}$ of all unary algebras $\left(X, f_{0}, f_{1}\right)$ is isomorphism universal (see [10] or [19]). Our aim is to represent every algebra from $2 \mathrm{~A}$ by an $h$-space dual to a Heyting algebra from $\mathbf{K}_{4}$. Similarly to the construction described in the preceding section, we first construct a locally compact ordered space $F\left(X, f_{0}, f_{1}\right)$ as follows.

Recall that subsets $U_{0}$ and $U_{1}$ of the subspace $B$ of $C$ were selected to satisfy $U_{k} \cap B_{i}=\left\{u_{2 i+k}\right\} \subseteq B_{i}(\omega)$ for all $i \geq 1$ and $k \in\{0,1\}$. Set $U=$ $U_{0} \cup U_{1}$, and let $U^{+}=\left\{u_{j}^{+}: j \geq 2\right\}$ be a copy of $U$ disjoint from $C$.

The underlying set of $F\left(X, f_{0}, f_{1}\right)$ is $S=X \times\left(C \cup U^{+}\right)$, and the order $\leq$ of the space $F\left(X, f_{0}, f_{1}\right)$ is given by

$$
(x, u) \leq\left(x, u^{+}\right) \text {for all } x \in X \text { and all } u \in U,
$$

and

$$
(x, c) \leq\left(x^{\prime}, c^{\prime}\right) \text { if and only if } x=x^{\prime} \text { and } c \leq c^{\prime} \text { in }(C, \leq) .
$$

Observe that every principal order ideal of $(S, \leq)$ is a chain with at most two elements, and that $X \times U^{+}$is an increasing subset of $S$.

Recall also that $s_{0}$ is the root of the underlying $\omega$-tree $W\left(s_{0}\right)$ of $B_{0}$, and that $B_{0} \backslash\left\{z_{0}\right\}$ is a subspace of $C$.

We intend to compactify each discrete space $f_{k}^{-1}\{x\} \times U_{k}^{+}$by the singleton $\left\{\left(x, s_{0}\right)\right\} \subseteq\{x\} \times C$ while conserving the topology of each $\{x\} \times C$ inherited from $C$.

More precisely, the topology $\sigma$ of $F\left(X, f_{0}, f_{1}\right)=(S, \sigma, \leq)$ is determined by requiring that $A \subseteq S$ be $\sigma$-open if and only if

(A) $\{c \in C:(x, c) \in A\}$ is $\gamma$-open for every $x \in X$,

(B) if $\left(x, s_{0}\right) \in A$ then $\left(f_{k}^{-1}\{x\} \times U_{k}^{+}\right) \backslash A$ is finite for $k \in\{0,1\}$.

Thus $\sigma$ is the union topology determined by all locally compact subspaces $\{x\} \times C$ (cf. 5.7) and by all compact spaces $D(x, k)=\left\{\left(x, s_{0}\right)\right\} \cup\left(f_{k}^{-1}\{x\} \times U_{k}^{+}\right)$ with $x \in X$ and $k \in\{0,1\}$. Clearly $D(x, 0) \cap D(x, 1)=\left\{\left(x, s_{0}\right)\right\}$, and $D(x, k) \cap D\left(x^{\prime}, k^{\prime}\right)=\varnothing$ for $x \neq x^{\prime}$. For every $x \in X$ set $D(x)=D(x, 0) \cup$ $D(x, 1)$.

For any $\gamma$-open $C^{\prime} \subseteq C$ such that $s_{0} \notin C^{\prime}$, the set $\{x\} \times C^{\prime}$ is $\sigma$ open, and so is the set $D(x) \cup\left(\{x\} \times C^{\prime \prime}\right)$ for any $\gamma$-open $C^{\prime \prime} \subseteq C$ with 
$s_{0} \in C^{\prime \prime}$. All singletons $\left\{\left(x, u^{+}\right)\right\} \subseteq X \times U^{+}$are $\sigma$-open. Thus any $\sigma$-open compact $R \subseteq S$ is disjoint from all but finitely many sets $\{x\} \times C$, the set $\left\{\left(x, u^{+}\right):\left(f_{k}(x), s_{0}\right) \notin R, u \in U_{k}\right\} \cap R$ is finite for $k \in\{0,1\}$ and, by 5.7, $C(x, R)=\{c \in C:(x, c) \in R\}$ is $\gamma$-clopen compact for each $x \in X$.

It is easily seen that a set $A \subseteq S$ is $\sigma$-clopen if and only if

(a) $\{c \in C:(x, c) \in A\}$ is $\gamma$-clopen for every $x \in X$,

(b) if $\left(x, s_{0}\right) \in A$ then $\left(f_{k}^{-1}\{x\} \times U_{k}^{+}\right) \backslash A$ is finite for $k \in\{0,1\}$, and

(c) if $\left(f_{k}^{-1}\{x\} \times U_{k}^{+}\right) \cap A$ is infinite for $k=0$ or $k=1$, then $\left(x, s_{0}\right) \in A$.

Lemma 5.9. The ordered space $F\left(X, f_{0}, f_{1}\right)=(S, \sigma, \leq)$ has the following two properties:

(a) the set $[R]$ is clopen compact for every clopen compact $R \subseteq S$, and

(b) if $s, t \in S$ and $s \mathbb{E} t$ then there exists a clopen compact increasing $R \subseteq S$ such that $s \in R$ and $t \in S \backslash R$.

Proof. We begin with an observation pertinent to both claims.

If $K$ is a $\gamma$-clopen compact subset of $C$ then, as noted in the proof of 5.8, $K \cap B$ is compact in $B$. Hence, by 5.6, $K$ intersects only finitely many subspaces $B_{i}$ of $B$. Since $B_{i} \cap\left(\left\{s_{0}\right\} \cup U\right)$ is finite for every $i \geq 0$, the set $K \cap\left(\left\{s_{0}\right\} \cup U\right)$ must be finite. Moreover, from 5.7 it follows that for every $c \in C$ there exists a $\gamma$-clopen compact increasing neighbourhood $K_{c} \subseteq C$ of $c$ such that $K_{c} \cap\left(\left\{s_{0}\right\} \cup U\right) \subseteq\{c\}$.

To prove (a), let $R \subseteq S$ be $\sigma$-clopen compact. There is a finite $F \subseteq X$ such that $C(x, R)=\{c \in C:(x, c) \in R\}=\varnothing$ for all $x \in X \backslash F$; since each $C(x, R)$ is $\gamma$-clopen compact, the set $C(x, R) \cap U$ is finite for every $x \in X$. If $s \in[R) \backslash R$ then there exists an $f \in F$ such that either $r=\left(f, y_{i}\right) \in R$ for some $i \geq 1$ and also $s \in\{f\} \times([C(f, R)) \backslash C(f, R)) \subseteq\{f\} \times C$, or else $r=(f, u)$ with $u \in U$ and $s=\left(f, u^{+}\right)$. The largest possible set $V=\left\{\left(f, u^{+}\right): u \in\right.$ $C(f, R) \cap U, f \in F\}$ of elements of the second kind is finite and clopen. Moreover, $[R) \backslash(R \cup V)=\bigcup\{\{f\} \times([C(f, R)) \backslash C(f, R)): f \in F\}$. Each of the finitely many sets $\{f\} \times([C(f, R)) \backslash C(f, R))$ is contained in $X \times(C \backslash B)$, and is $\gamma$-clopen compact by 5.8; hence it is also $\sigma$-clopen. Thus $[R) \backslash(R \cup V)$ is $\sigma$-clopen and compact. Therefore $[R]$ is a union of finitely many $\sigma$-clopen compact sets.

To verify (b), suppose that $s \not t$ in $S$.

If $s=\left(x, u^{+}\right)$for some $u \in U$ then $R=[\{s\})=\{s\}$ is clopen compact and $t \in S \backslash R$.

Let $s=(x, c)$ for some $c \in C \backslash\left(\left\{s_{0}\right\} \cup U\right)$. If $K_{c}$ is as defined in the first paragraph of this proof, then the set $\{x\} \times K_{c}$ is $\sigma$-clopen compact, and $\left[\{x\} \times K_{c}\right)=\{x\} \times K_{c}$ in $(S, \leq)$. If $t \in\{x\} \times K_{c}$ then $t=(x, d)$ for some $d \in C$ such that $c \notin d$, and there is a $\gamma$-clopen compact increasing $K \subseteq C$ containing $c$ but not $d$. The set $R=\{x\} \times\left(K_{c} \cap K\right)$ is $\sigma$-clopen compact and increasing as required. 
For $s=\left(x, s_{0}\right)$, the set $D=D(x) \cup\left(\{x\} \times K_{s_{0}}\right)$ is a $\sigma$-clopen compact increasing neighbourhood of $\left(x, s_{0}\right)$ disjoint from $\{x\} \times U$. If $t \in D$ then either $t=(x, d)$ for some $d \in C$ such that $s_{0} \leq d$ and we select $K$ as in the previous case and set $R=D(x) \cup\left(\{x\} \times\left(K_{s_{0}} \cap K\right)\right)$, or else $t \in X \times U^{+}$and we choose $R=D \backslash\{t\}$.

If $s=(x, u)$ for some $u \in U$ then the set $\left[\{x\} \times K_{u}\right)$ is $\sigma$-clopen compact increasing, and $\left[\{x\} \times K_{u}\right) \backslash\{x\} \times K_{u}=\left\{\left(x, u^{+}\right)\right\}$. If $t \in\left[\{x\} \times K_{u}\right)$ then $t \neq\left(x, u^{+}\right)$by hypothesis; thus $t=(x, d)$ for some $d \geq u$ in $C$. Again by 5.7, there is a $\gamma$-clopen compact increasing $K \subseteq C$ such that $u \in K$ and $d \in C \backslash K$, and we set $R=\left[\{x\} \times\left(K \cap K_{u}\right)\right)$.

Finally, the ordered space $\Phi\left(X, f_{0}, f_{1}\right)$ representing an algebra $\left(X, f_{0}, f_{1}\right)$ $\in 2 \mathbf{A}$ is defined as a one-point compactification of $(S, \sigma, \leq)=F\left(X, f_{0}, f_{1}\right)$ by a least element 0 . The resulting partial order is a tree rooted at 0 whose chains have at most three elements. Once it is shown that $(S, \sigma, \leq)$ is an $h$-space, it will thus also be proved that it represents an algebra from $\mathbf{K}_{4}$.

All $\sigma$-clopen compact (increasing) sets remain clopen (increasing) in the compact space $\Phi\left(X, f_{0}, f_{1}\right)$. Thus $\Phi\left(X, f_{0}, f_{1}\right)$ is a Priestley space by $5.9(\mathrm{~b})$. Since $\{0\}$ compactifies $F\left(X, f_{0}, f_{1}\right)$ in $\Phi\left(X, f_{0}, f_{1}\right)$, each clopen set $D$ not containing the least element 0 is $\sigma$-clopen compact; thus $[D)$ is $\sigma$-clopen compact by $5.9(\mathrm{a})$, and hence a clopen increasing subset of $\Phi\left(X, f_{0}, f_{1}\right)$.

We summarize these remarks as follows.

Proposition 5.10. $\Phi\left(X, f_{0}, f_{1}\right)$ is an h-space dual to an algebra in $\mathbf{K}_{4}$.

For any isomorphism $g:\left(X, f_{0}, f_{1}\right) \rightarrow\left(X^{\prime}, f_{0}, f_{1}\right)$ in 2A define

$$
\Phi(g):\{0\} \cup X \times\left(C \cup U^{+}\right) \rightarrow\{0\} \cup X^{\prime} \times\left(C \cup U^{+}\right)
$$

by

$$
\begin{gathered}
\Phi(g)(x, p)=(g(x), p) \text { for all } x \in X \text { and all } p \in C \cup U^{+}, \\
\Phi(g)(0)=0 .
\end{gathered}
$$

Lemma 5.11. If $g$ is an isomorphism in $2 \mathbf{A}$ then $\Phi(g)$ is a homeomorphism and order isomorphism.

Proof. The restriction of $\Phi(g)$ to $\{x\} \times C$ is clearly an order homeomorphism onto $\{g(x)\} \times C$ for each $x \in X$. Since $(x, u) \leq\left(x, u^{+}\right)$implies $(g(x), u) \leq$ $\left(g(x), u^{+}\right)$, the mapping $\Phi(g)$ is an order isomorphism. From $f_{k} \circ g=g \circ f_{k}$ for $k \in\{0,1\}$ we obtain $g^{-1}\left(f_{k}^{-1}\{x\}\right)=f_{k}^{-1}\left\{g^{-1}(x)\right\}$ and, consequently,

$$
\begin{aligned}
\Phi(g)^{-1}(D(x, k)) & =\Phi(g)^{-1}\left(\left\{\left(x, s_{0}\right)\right\} \cup\left(f_{k}^{-1}\{x\} \times U_{k}^{+}\right)\right) \\
& =\left\{\left(g^{-1}(x), s_{0}\right)\right\} \cup\left(f_{k}^{-1}\left\{g^{-1}(x)\right\} \times U_{k}^{+}\right) \\
& =D\left(g^{-1}(x), k\right) \text { for } k \in\{0,1\} .
\end{aligned}
$$

Therefore $\Phi(g)^{-1}(A)$ is $\sigma$-open for every $\sigma$-open $A$, and the conclusion follows. 
Lemma 5.12. If $h: \Phi(A) \rightarrow \Phi\left(A^{\prime}\right)$ is a homeomorphism and an order isomorphism then there exists a unique isomorphism $g: A \rightarrow A^{\prime}$ in $2 \mathbf{A}$ such that $h=\Phi(g)$.

Proof. Since 0 is the unique minimal element in both spaces, $h(0)=0$ is immediately obtained.

The set $X \times U^{+}$is the set of all open points of $\Phi(A)$; hence $h\left(X \times U^{+}\right)=$ $X^{\prime} \times U^{+}$and, consequently, $h(X \times C)=X^{\prime} \times C$.

Furthermore, $X \times\left\{y_{1}, y_{2}, \ldots\right\}$ is the set of all nonmaximal members of $X \times C$; as a result, $h\left(x, y_{i}\right)=\left(x^{\prime}, y_{j}\right)$ for some $x^{\prime} \in X^{\prime}$ and $j \in\{1,2, \ldots\}$. Elements strictly above $\left(x, y_{i}\right)$ form a subspace $\{x\} \times T \times\{i\}$ homeomorphic to the heterogeneous tree space $(T, \tau)$, so that $h\left(x, y_{i}\right)=\left(x^{\prime}, y_{j}\right)$ implies $h(x, t, i)=\left(x^{\prime}, t, j\right)$ for every $t \in T$ by 5.3. Being invertible, $h$ maps $X \times T \times\{1,2, \ldots\}$ bijectively onto $X^{\prime} \times T \times\{1,2, \ldots\}$. Since $X \times B$ is the complement of the latter set in $X \times C$, we obtain $h(X \times B)=X^{\prime} \times B$.

For any $x \in X$ and $t \in T$ set $S(x, t)=\{(x, t, i): i \geq 1\}$. Recall that for $t=b \in B$, the closure $\operatorname{cl}(S(x, b))=S(x, b) \cup\{(x, b)\}$. If $h(x, b)=\left(x^{\prime}, b^{\prime}\right)$ then $\left(x^{\prime}, b^{\prime}\right)$ is the only closure point of $h(S(x, b))=\{h(x, b, i): i \geq 1\}$ outside this set. This is possible only when the set

$$
I=\left\{i \geq 1:(\exists j \geq 1) h(x, b, i)=\left(x^{\prime}, b, j\right)\right\}
$$

is infinite and $b^{\prime}=b$. But then $h\left\{\left(x, y_{i}\right): i \in I\right\}=\left\{\left(x^{\prime}, y_{j}\right): j \in I^{\prime}\right\}$ and $h(\{x\} \times T \times I)=\left\{x^{\prime}\right\} \times T \times I^{\prime}$, where $I^{\prime}=\left\{j:(\exists i \in I) h\left(x, y_{i}\right)=\left(x^{\prime}, y_{j}\right)\right\}$. Recalling that $\{x\} \times B \subseteq \operatorname{cl}(\{x\} \times T \times I)$ for any infinite set $I$, we now conclude that $h(\{x\} \times B) \subseteq \operatorname{cl}\left(\left\{x^{\prime}\right\} \times T \times I^{\prime}\right) \subseteq\left\{x^{\prime}\right\} \times C$. Consequently, $h(x, b)=\left(x^{\prime}, b\right)$ for all $b \in B$.

In particular then $h\left(x, y_{i}\right)=\left(x^{\prime}, y_{i}\right)$ for every $i \geq 1$; hence $h(x, t, i)=$ $\left(x^{\prime}, t, i\right)$ for all $t \in T$ and $i \geq 1$. Thus we can define a bijection $g$ of $X$ onto $X^{\prime}$ such that $h(x, c)=(g(x), c)$ for all $(x, c) \in X \times C$.

Every $\left(x, u^{+}\right) \in X \times U^{+}$is a unique cover of the element $(x, u) \in X \times$ $U \subseteq X \times C$, and $\left(\left(x, u^{+}\right)\right]=\left\{0,(x, u),\left(x, u^{+}\right)\right\}$. Therefore $h\left(x, u^{+}\right)=$ $\left(g(x), u^{+}\right)$, and $h=\Phi(g)$ is obtained. It remains to show that $g$ is an isomorphism of $A$ onto $A^{\prime}$.

Recall that $\left(y, s_{0}\right)$ is the only limit point of the discrete set $f_{k}^{-1}\{y\} \times U_{k}^{+}$ for each $y \in X$ and $k \in\{0,1\}$. Since the set $\{x\} \times U_{k}^{+}$is infinite for every $x \in X$, its limit point is $\left(f_{k}(x), s_{0}\right)$. Therefore $h\left(f_{k}(x), s_{0}\right)=\left(g\left(f_{k}(x)\right), s_{0}\right)$ is the limit point of $h\left(\{x\} \times U_{k}^{+}\right)=\{g(x)\} \times U_{k}^{+}$, namely the point $\left(f_{k}(g(x)), s_{0}\right)$. Thus $g\left(f_{k}(x)\right)=f_{k}(g(x))$ for every $x \in X$ and $k \in\{0,1\}$; in other words, $g$ is the isomorphism in $2 \mathbf{A}$ for which $\Phi(g)=h$.

The proof of Theorem 1.1 is now complete.

\section{CONCLUding REMARKS}

The varieties $\mathbf{H}, \mathbf{H}^{\prime}$, and all $\mathbf{K}_{n}$ with finite $n \geq 4$ are examples of finitely generated congruence permutable isomorphism universal varieties. Their exis- 
tence provides a partial answer to Problem 1 of [13], which is a modification of an unpublished question posed by Ervin Fried. Are there any other such examples?

It may be of some interest to note that, in contrast to Heyting algebras, finitely generated isomorphism universal varieties of distributive double $p$-algebras coincide with those which are not congruence permutable [13].

Varieties $\mathbf{K}_{n}$ of relative Stone algebras which are not isomorphism universal coincide with those on which Davey's duality is full [5], and also with those generated by an injective algebra (see Davey [5] and Day [6]). This coincidence is perhaps somewhat spurious, for Davey's duality is full also on the injectivegenerated variety of all double Stone algebras which is isomorphism universal [13].

\section{REFERENCES}

1. M. E. Adams, V. Koubek, and J. Sichler, Homomorphisms and endomorphisms in varieties of pseudocomplemented distributive lattices (with applications to Heyting algebras ), Trans. Amer. Math. Soc. 285 (1984), 57-79.

2. R. Balbes and Ph. Dwinger, Distributive lattices, Univ. of Missouri Press, Columbia, Mo., 1974.

3. G. Birkhoff, On groups of automorphisms, Rev. Un. Mat. Argentina 11 (1946), 155-157.

4. G. Brenner, A simple construction for rigid and weakly homogeneous Boolean algebras answering a question of Rubin, Proc. Amer. Math. Soc. 87 (1983), 601-606.

5. B. A. Davey, Dualities for Stone algebras, double Stone algebras and relative Stone algebras, Colloq. Math. 46 (1982), 1-14.

6. Alan Day, Injectivity in equational classes of algebras, Canad. J. Math. 24 (1972), 209-220.

7. R. Frucht, Herstellung von Graphen mit vorgegebener abstrakten Gruppe, Compositio Math. 6 (1938), 239-250.

8. G. Gierz, K. H. Hofmann, K. Keimel, J. D. Lawson, M. Mislove, and D. S. Scott, A compendium of continuous lattices, Springer-Verlag, Berlin, Heidelberg and New York, 1980.

9. T. Hecht and T. Katriňák, Equational classes of relative Stone algebras, Notre Dame J. Formal Logic 13 (1972), 248-254.

10. Z. Hedrlín and A. Pultr, On full embeddings of categories of algebras, Illinois J. Math. 10 (1966), 392-406.

11. V. A. Jankov, Constructing a sequence of strongly independent superintuitionistic propositional calculi, Soviet Math. Dokl. 9 (1968), 806-807.

12. J. L. Kelley, General topology, Van Nostrand Reinhold, New York, 1955.

13. V. Koubek and J. Sichler, Universal varieties of distributive double p-algebras, Glasgow J. Math. 26 (1985), 121-131.

14. R. McKenzie and J. D. Monk, On automorphism groups of Boolean algebras, Infinite and Finite Sets (Colloq., Keszthely, 1973; dedicated to P. Erdös on his 60th birthday), Vol. II, Collog. Math. Soc. Jámos Bolyai, Vol. 10, North-Holland, Amsterdam, 1975, pp. 951-988.

15. J. Nešetřil and A. Pultr, Representing monoids by mappings preserving equivalences, Algebra Universalis 1 (1971), 155-162.

16. H. A. Priestley, Representation of distributive lattices by means of ordered Stone spaces, Bull. London Math. Soc. 2 (1970), 186-190.

17. __ Ordered topological spaces and the representation of distributive lattices, Proc. London Math. Soc. (3) 24 (1972), 507-530. 
18. __ Ordered sets and duality for distributive lattices, Ann. Discrete Math. 23 (1984), 3960.

19. A. Pultr and V. Trnková, Combinatorial, algebraic and topological representations of groups, semigroups and categories, North-Holland, Amsterdam, 1980.

20. J. Sichler, Group universal unary varieties, Algebra Universalis 11 (1980), 12-21.

Department of Mathematics, State University of New York, New Paltz, New York 12561

MFF KU, Malostranské NáM. 25, 11800 Praha 1, Czechoslovakia

Department of Mathematics and Astronomy, University of Manitoba, WinNipeg, MaNITOBA, CANADA R3T 2N2 